\title{
A POSITRON SOURCE USING AN AXIALLY ORIENTED CRYSTAL ASSOCIATED TO A GRANULAR AMORPHOUS CONVERTER
}

\author{
Chenghai Xu ${ }^{1,2}$, Robert Chehab $^{3}$, Peter Sievers ${ }^{4}$, Xavier Artru ${ }^{3}$, Michel Chevallier ${ }^{3}$ \\ Olivier Dadoun ${ }^{2}$,GuoxiPei ${ }^{1}$, Vladimir M.Strakhovenko ${ }^{5}$,AlessandroVariola ${ }^{2},{ }^{, 1}$ IHEP, \\ Beijing, China, ${ }^{2}$ LAL, CNRS/IN2P3, Orsay, France, ${ }^{3}$ IPNL, CNRS/IN2P3, Lyon, France, \\ ${ }^{4}$ CERN, Geneva, Switzerland, ${ }^{5}$ BINP, Novosibirsk, Russia
}

\begin{abstract}
A non conventional positron source using the intense $\gamma$ radiation from an axially oriented monocrystal which materialize into e+e- pairs in a granular amorphous converter is described. The enhancement of photon radiation by multi-GeV electrons crossing a tungsten crystal along its $<111>$ axis is reported. The resulting enhancement of pair production in an amorphous converter placed 2 meters downstream, is also reported. Sweeping off the charged particles from the crystal by a bending magnet upstream of the converter allows a significant reduction of the deposited energy density. Substituting a granular target made of small spheres to the usual compact one, makes easier the energy dissipation. The deposited energy and corresponding heating are analyzed and solutions for cooling are proposed. The configurations studied here for this kind of positron source allow its consideration for unpolarized positrons for the ILC.
\end{abstract}

\section{1- INTRODUCTION}

The use of axially oriented crystals with GeV electron beams in channeling conditions provide powerful sources of (unpolarized) photons. These photons can, then, materialize into e-e+ pairs in the same crystal or in amorphous converters put downstream. Theoretical studies [1] and simulations [2-4] were confirmed by experiments at CERN and KEK [5-9]. Separating the crystal-radiator from the amorphous-converter with a bending magnet in between to sweep off the charged particles, allowing only photons to impinge on the converter, presents very interesting features concerning the amount of energy deposited and for its density. [10]. For such device, called hybrid source, the amount of PEDD (Peak Energy Deposition Density) can be made lower than critical values ( $35 \mathrm{~J} / \mathrm{g}$, for $\mathrm{W}$ ) avoiding the use ofa multitarget system; that was already proposed and adopted for CLIC for its baseline [11]. However, the problem of very intense beams as for ILC may present a serious problem for the target survival. We have carried out a study on a particular kind of converter made of granular substance (W spheres) which present the advantages of easier thermal dissipation; it was already considered for targets submitted to high intensity proton beams and dedicated to neutrino factories (P.Sievers, [12]) . Some results concerning a hybrid target with a granular converter are presented here.

\section{2- THE HYBRID SOURCE}

A high energy electron beam ( 5 to $10 \mathrm{GeV}$ ) is directed along the crystal axis . Photons, electrons and positrons are generated. A sweeping magnet takes off the charged particles and only the photons impinge on the amorphous converter (figure 1). The distance may be about 2 meters. For the ILC, we consider an electron beam of $10 \mathrm{GeV}$ with a transverse rms radius of $2.5 \mathrm{~mm}$. A scheme of the hybrid source is presented on figure 1. 


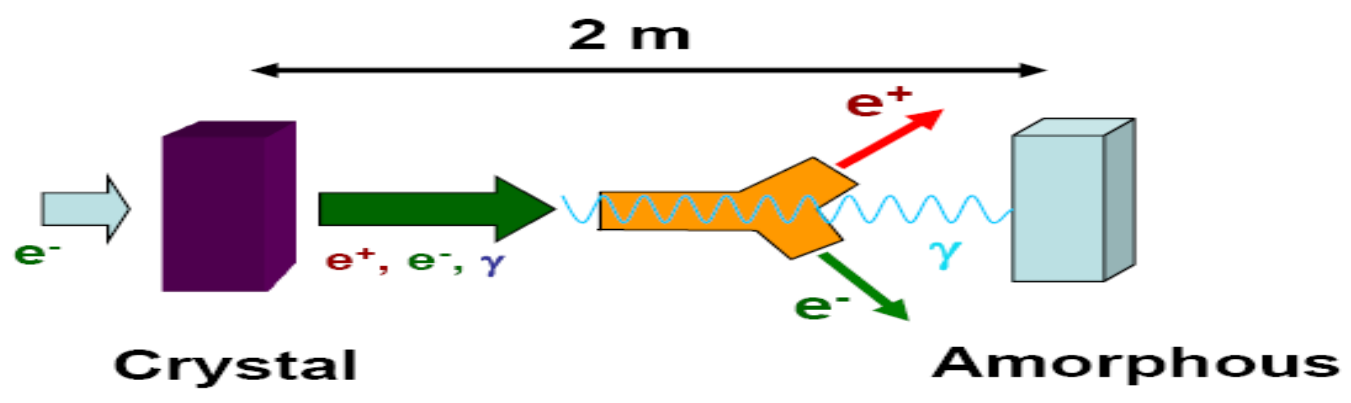

Fig. 1 The hybrid positron source

\section{2-1 The crystal radiator}

The idea to substitute an oriented crystal to the magnetic undulator in order to produce an intense source of photons was generated more than twenty years ago [13 ]. Two main arguments prevailed: the very strong fields available on the atomic rows of the crystal, equivalent to $10^{3}$ Tesla and the short periods associated to the oscillating trajectories of the electrons along the crystal axes $(\sim 1 \mu \mathrm{m})$. The latter characteristics allows to reach tens of $\mathrm{Mev}$ photon energy with a $\mathrm{GeV}$ electron beam incident on the crystal; that represents two orders of magnitude less energy than for an undulator having $\mathrm{cm}$ period..

The energy spectrum and the yield of the photons produced in the crystal depend, obviously, on the electron incident energy. For that, it is interesting to consider a figure of merit [1] given by the ratio:

$$
\mathrm{R}=\mathrm{I}_{\mathrm{ch}} / \mathrm{I}_{\mathrm{br}}
$$

where $\mathrm{I}_{\mathrm{ch}}$ is the intensity of channeling radiation and $\mathrm{I}_{\mathrm{br}}$, that of bremsstrahlung. This ratio depends on the electron energy and on the crystal. For instance, $\mathrm{R}=1$ for the axis $\langle 111\rangle$ at 0.7 $\mathrm{GeV}$ for $\mathrm{W}, 1.3 \mathrm{GeV}$ for $\mathrm{Si}$ and $1.9 \mathrm{GeV}$ for $\mathrm{Ge}$. At much higher energies, $\mathrm{R}>>1$ and the crystal effects dominate on bremsstrahlung and $\mathrm{R}$ is growing very fast with the energy. The radiation yield depends also of the crystal thickness. Increasing the crystal thickness, one may meet saturation due to the multiple scattering and the decrease in energy of the radiating particles.

Another peculiarity of the channeling radiation is that the emitted photons are much softer in energy than those created by bremsstrahlung. Therefore, the channeling radiation may be more efficient than bremsstrahlung, even with $\mathrm{R}=1$, providing a larger number of photons. For the application foreseen here, the ILC positron source, we have chosen an incident energy of $10 \mathrm{GeV}$ and a W crystal, with $<111>$ axial orientation and $1 \mathrm{~mm}$ thickness. To fulfill good channeling operation, the following conditions are required:

- A mosaic spread lower than the channeling critical angle

- An incident beam divergence, lower than the channeling critical angle.

This critical angle is given by :

$\psi_{\mathrm{c}}=[2 \mathrm{Uo} / \mathrm{E}]^{1 / 2}$

whereUo is the depth of the potential well created by the atomic rows and $\mathrm{E}$ the electron energy. For our conditions $(10 \mathrm{GeV},<111>\mathrm{W}$ axis) the critical angle is $\sim 0.5 \mathrm{mrad}$. 


\section{2-2 The granular converter}

The very intense incident electron beam considered in the linear collider projects $\left(10^{14}\right.$ e-/second) requiresresistant targets. In the muon collider and neutrino factories projects, a similar situation is met with powerful proton beams impinging on targets to produce pions decaying intomuons and then into neutrinos. P.Sievers and P. Pugnat have proposed the use of granular targets made of a great quantity of small spheres [12]. The high ratio of surface/volume of the spheres $(\sim 3 / \mathrm{r})$ makes easier the thermal dissipation. In the scheme considered for the converter of the hybrid source, the spheres are arranged in staggered rows. An example is shown on figure 2.

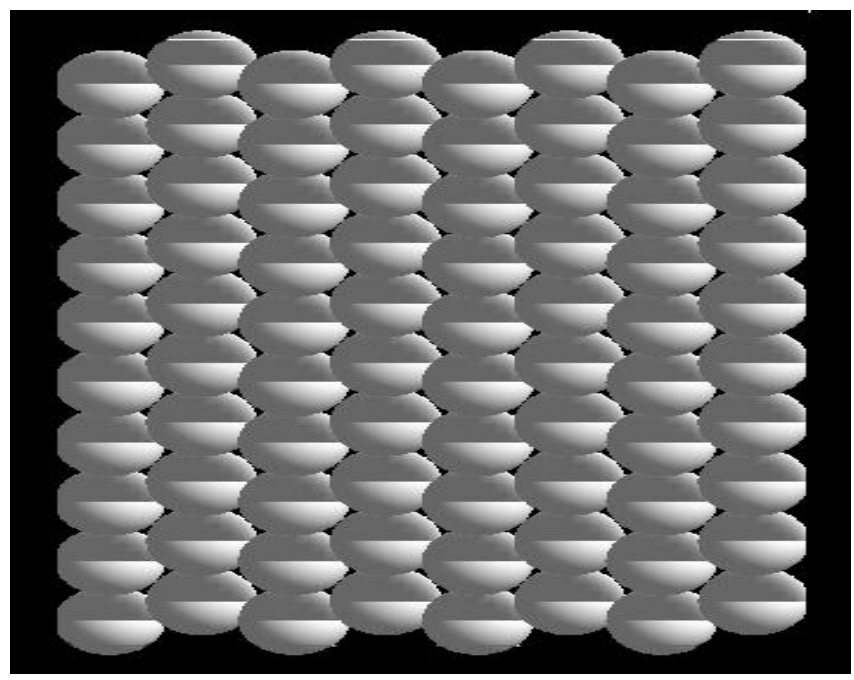

Fig. 2 A Granular target : 8 rows of $\mathrm{r}=1 \mathrm{~mm}$ spheres.

The choice of the number of rows is related to two essential quantities:

- The required positron yield

- $\quad$ The Peak Energy Deposition Density (PEDD)

For that purpose, we have made a comparison of two choices of granular targets ( $\mathrm{r}=1$ $\mathrm{mm}$ and $\mathrm{r}=0.5 \mathrm{~mm}$ ) with a compact target $8 \mathrm{~mm}$ thick which was convenient for the required positron yield. This comparison is shown on table 1 .

\begin{tabular}{|l|l|l|l|l|l|l|l|}
\hline & thickness & yield & PEDD & $(\Delta$ E)dep & N-layers & $\begin{array}{l}\text { spheres } \\
\text { number }\end{array}$ & $\begin{array}{l}\text { Effective } \\
\text { density }\end{array}$ \\
\hline unity & $\mathrm{mm}$ & $\mathrm{e}+/ \mathrm{e}-$ & $\begin{array}{l}\text { GeV/cm } \\
\text { e- }\end{array}$ & MeV/e- & & & g.cm $^{-3}$ \\
\hline compact & 8 & 13.3 & 2.24 & 523 & & & 19.3 \\
\hline $\begin{array}{l}\text { Granular } \\
\text { r=1 mm }\end{array}$ & 10.16 & 12.5 & 1.8 & 446 & 3 & 864 & 13.9 \\
\hline $\begin{array}{l}\text { Granular } \\
\text { r= 0.5mm }\end{array}$ & 11.60 & 13.45 & 2.33 & 613 & 7 & 8064 & 13.9 \\
\hline
\end{tabular}

Table 1. Comparison of compact and granular targets 
We can observe that the "3 layers" granular target has almost $6 \%$ less positron yield than the compact target but $20 \%$ lower PEDD. It looks more interesting from that point of view than the $\mathrm{r}=0.5 \mathrm{~mm}$ granular solution. These considerations may also be derived from figure 3, where the total yield and energy deposited normalized to the incident electron are reported in function of the target thickness expressed in $\mathrm{mm}$ or in number of layers.

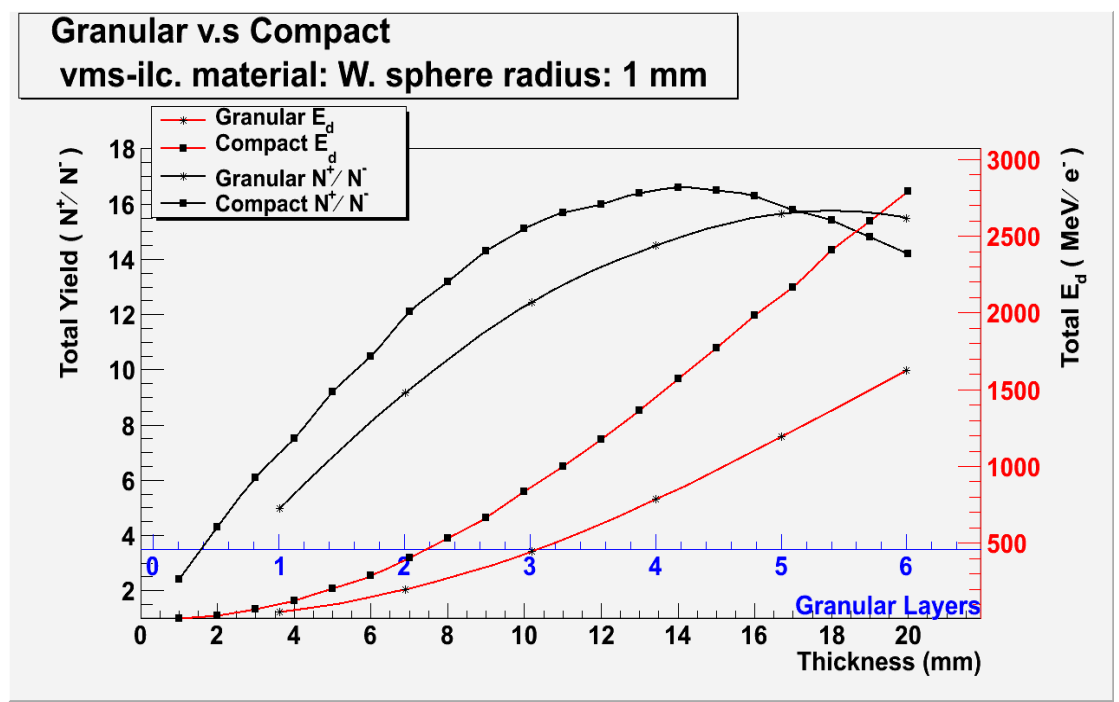

Fig.3 Comparison of compact and granular targets

We can see that the deposited energy for the "3 layers" target is $446 \mathrm{MeV} / \mathrm{e}$ - whereas it is of $523 \mathrm{MeV} / \mathrm{e}$ - for the $8 \mathrm{~mm}$ compact target.

\section{3- THE SIMULATIONS}

The simulations have been operated with twoprogrammes:

- A programme(VMS) describing the crystal effects (channeling, coherent bremsstrahlung, pair creation,..) written by V.M.Strakhovenko [ 14].

- The GEANT4 code which uses the ouputs of the first programme as event generator; results on positron production are provided by GEANT4.

Crystal effects are also described by the programme FOT; the results agree with VMS..

\section{3-1 The results on photons from the crystal}

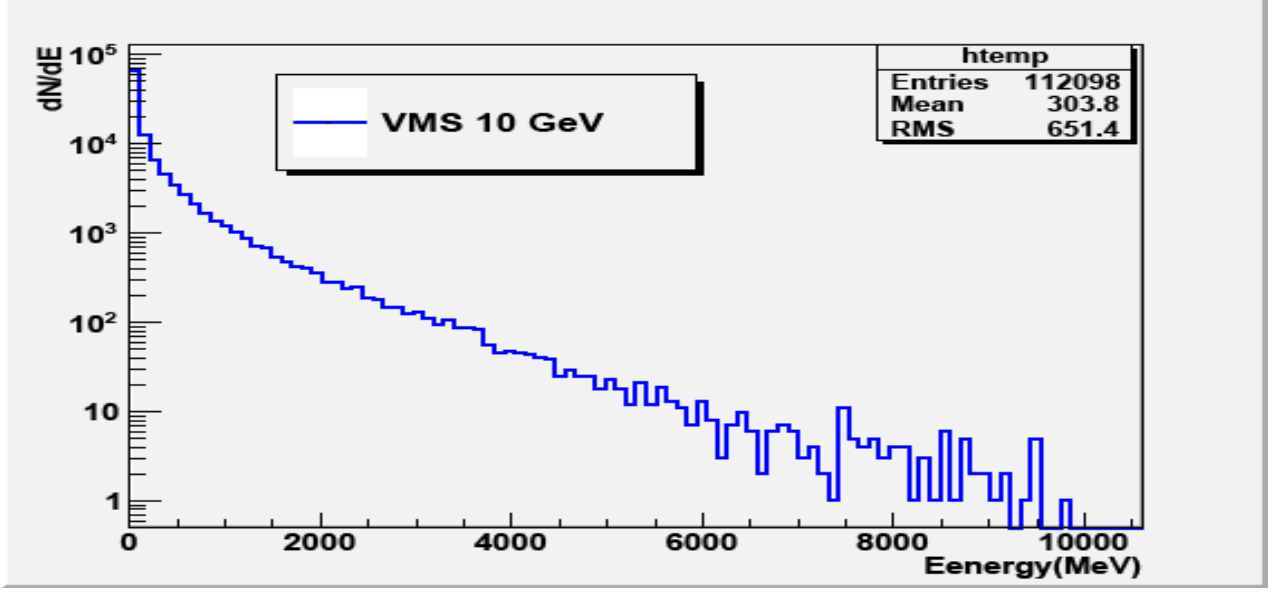

Fig. 4 The photon energy spectrum $[\mathrm{E}-=10 \mathrm{GeV} ; \mathrm{l}=1 \mathrm{~mm} \mathrm{~W}$ crystal $]$ 
The photon characteristics are obviously important for the positron generation. We represent on figure 4 the energy spectrum in the case of a $10 \mathrm{GeV}$ incident electron beam on a $1 \mathrm{~mm}$ thick crystal target oriented on its $\langle 111\rangle$ axis. On figure 5, we have represented the transverse characteristics of the photon beam impinging on the granular converter. These photons have been created by an electron beam having an rms radius of $2.5 \mathrm{~mm}$ and an energy of $10 \mathrm{GeV}$. The rms photon beam radius at the target is $\sim 3.5 \mathrm{~mm}$.

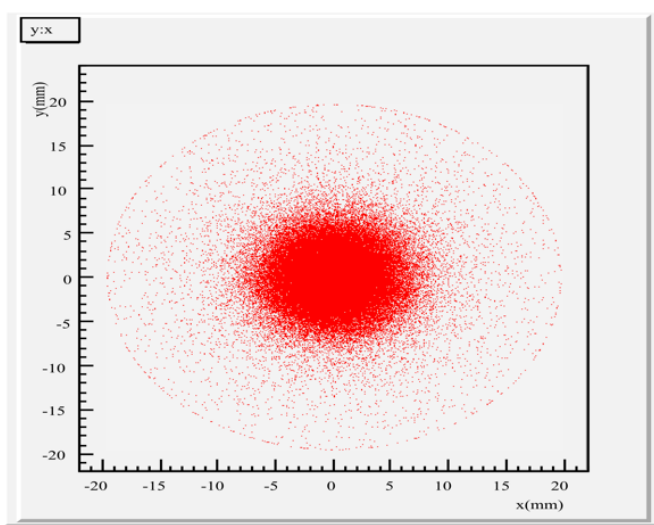

Fig.5-a

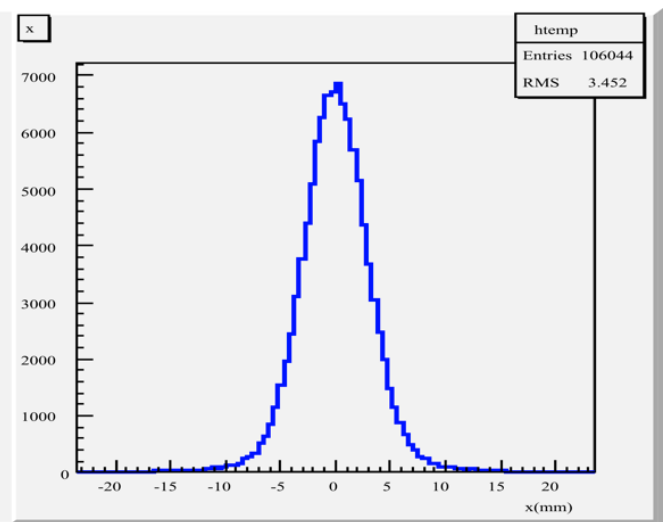

Fig.5-b

Fig.5The transverse characteristics of the photon beam at the converter; (a) x-y distribution; (b) Marginal y distribution on $\mathrm{x}$ plane; beam dimensions are given in $\mathrm{mm}$.

\section{3-2 Simulation results on positrons}

\section{3-2-1 The positron beam}

The positrons created in the granular target (3 layers) are captured by an Adiabatic Matching device (AMD) which exhibits a slowly decreasing axial magnetic field from the converter to the entrance of the first accelerating section. The field is tapering from 6 tesla to 0.5 tesla over $50 \mathrm{~cm}$. We present on figure 6 the energy spectrum, transverse dimension and momentum and time distribution at the converter exit. The same kind of figure (figure 7) concernes the same parameters after the capture by the AMD and one meter acceleration. We can observe the phase space transformation due to the matching device converting the small dimensions and large transverse momentum at the converter into larger dimensions and smaller transverse momentum after the AMD and one meter acceleration.
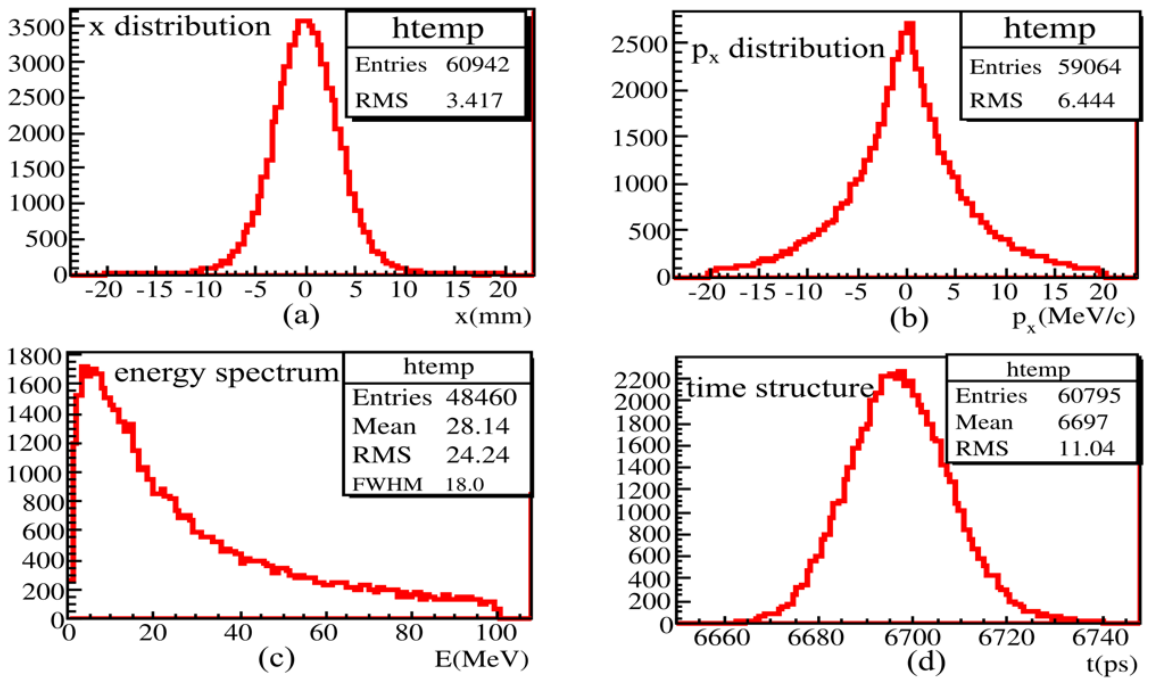

Fig.6 Transverse dimensions and momentum, energy spectrum and time distribution at the target exit 

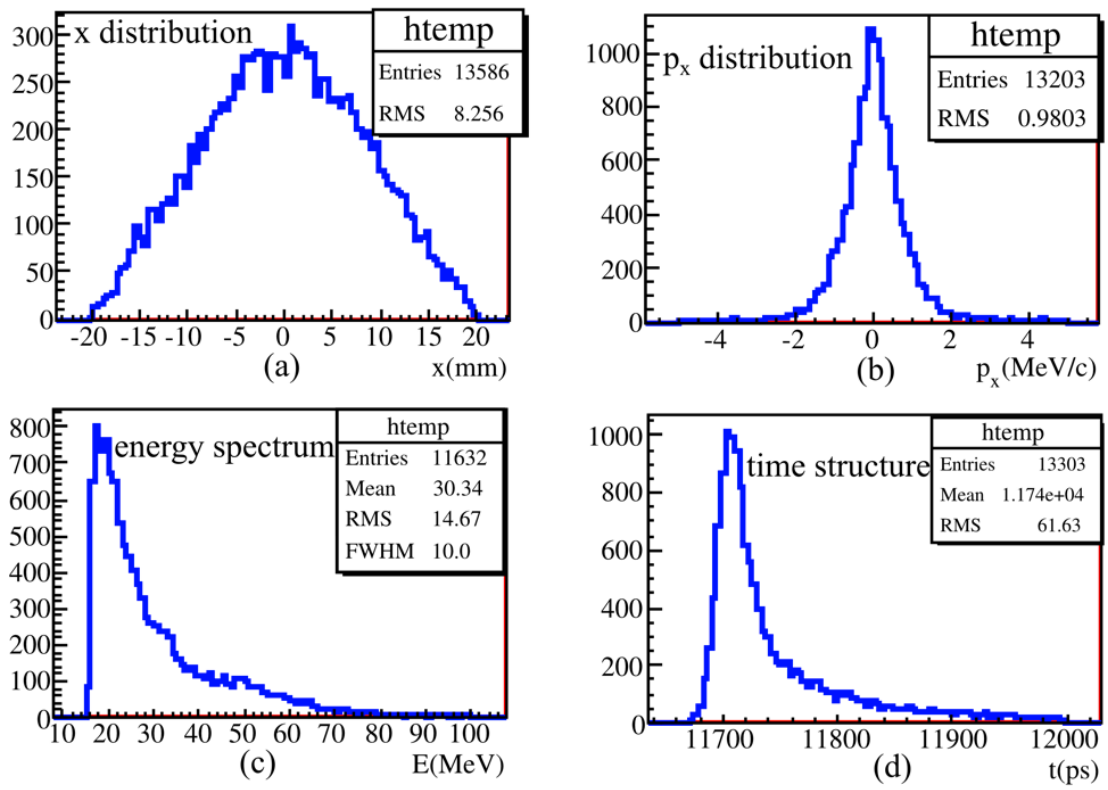

Fig. 7 Transverse dimensions and momentum, energy spectrum and time distribution after acceleration

\section{3-2-2 Energy deposited and PEDD}

The configuration chosen for the linear collider ILC is based on a pulse time structure modification before the target in order to decrease the power deposited per pulse; the nominal structure of ILC beam is then recuperated after the Damping Ring. For that purpose, we have chosen the configuration proposed by T.Omorifrom KEK [15] and where the incident electron beam is made of minitrains of 100 bunches each with a periodicity of $300 \mathrm{~Hz}$ in a macropulse of $40 \mathrm{~ms}$ containing 13 minitrains. See figure 8.

\section{$300 \mathrm{~Hz}$ Advanced Conventional $\mathrm{e}^{+}$Source for MM Crystal/Amorphous Hybrid Target \\ Normal Conducting Drive and Booster Linacs in $300 \mathrm{~Hz}$ operation}

e+ creation

100 bunches/train $\times 300 \mathrm{~Hz}$

$\mathrm{T}_{\mathrm{b} \_ \text {to } b}=6.15 \mathrm{n} \mathrm{sec}$ go to main linac

1300 bunches/train $\times 5 \mathrm{~Hz}$

$\mathrm{T}_{\mathrm{b} \text { to } \mathrm{b}}=\mathbf{3 0 0 \mathrm { n } \text { sec }}$

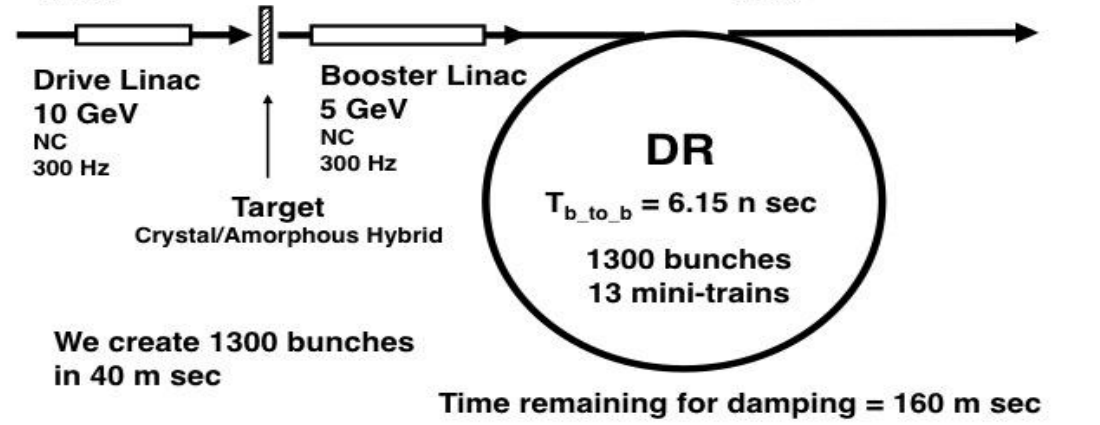

Fig. 8The scheme of the $300 \mathrm{~Hz}$ solution proposed by Omori et al.

The first target submitted to the incident beam is the crystal; An estimation of the energy deposited and of the PEDD is necessary. 
The $10 \mathrm{GeV}$ electron beam with an rms beam radius of $2.5 \mathrm{~mm}$ deposit about $8 \mathrm{MeV} / \mathrm{e}$ - in the $1 \mathrm{~mm}$ thick $\mathrm{W}$ crystal. That represents a power of 160 watts. Cooling is foreseen. The energy deposition density has a peak (PEDD) of $0.35 \mathrm{GeV} / \mathrm{cm} 3 / \mathrm{e}-.$. That represents for the ILC beam a PEDD of $5.8 \mathrm{~J} / \mathrm{g}$ for a minitrain of $2.10^{12}$ impinginge-. The minitrains are separated by $3.3 \mathrm{~ms}$, giving enough time to the relaxation of shock waves.

Another problem for the crystal concernsthe high rate of Coulomb scattering on the nuclei which could lead to dislodgements and affect the crystal structure. The fluence is $2.10^{16} \mathrm{e}-/ \mathrm{mm}^{2} /$ hour. It needs about 100 hours to reach the fluenceobtained in the SLAC test in 1997 where no damage was observed on the crystal [16]. Estimating the critical fluence one order of magnitude larger, leads to a working time of thousand hours without estimated damage. Annealing procedures helps to restore the crystal qualities.

The crystal must be cooled. It can be mounted in a water cooled round frame (a) (radially constrained) or mounted on only one side (with verticalbut no horizontal expansion. The target unit (Crystal + Be-windows) could also be cooled by a He jet (b), the heat being evacuated through both sides faces of the crystal. This allows to devise a support for the crystal which provides the required stability in its orientation, but permits its unconstrained lateral thermal expansion, to reduce thermal stresses and fatigue.Fivecrystals can be put on a transversally movingframe: that will reduce the average power deposited whereas the PEDD per micropulse $(0.6 \mu \mathrm{s})$ and per macropulse $(40 \mathrm{~ms})$ will not change. The fluence associatedwith radiation damages is decreased by a factor 5 . This choice is compatible with the use of a goniometer in which a horizontal motion is foreseen withthe two rotations ( $\mathrm{H}$ and $\mathrm{V}$ axes) .The device is shown on figure 9. The translation direction is perpendicular to the plane of the figure.

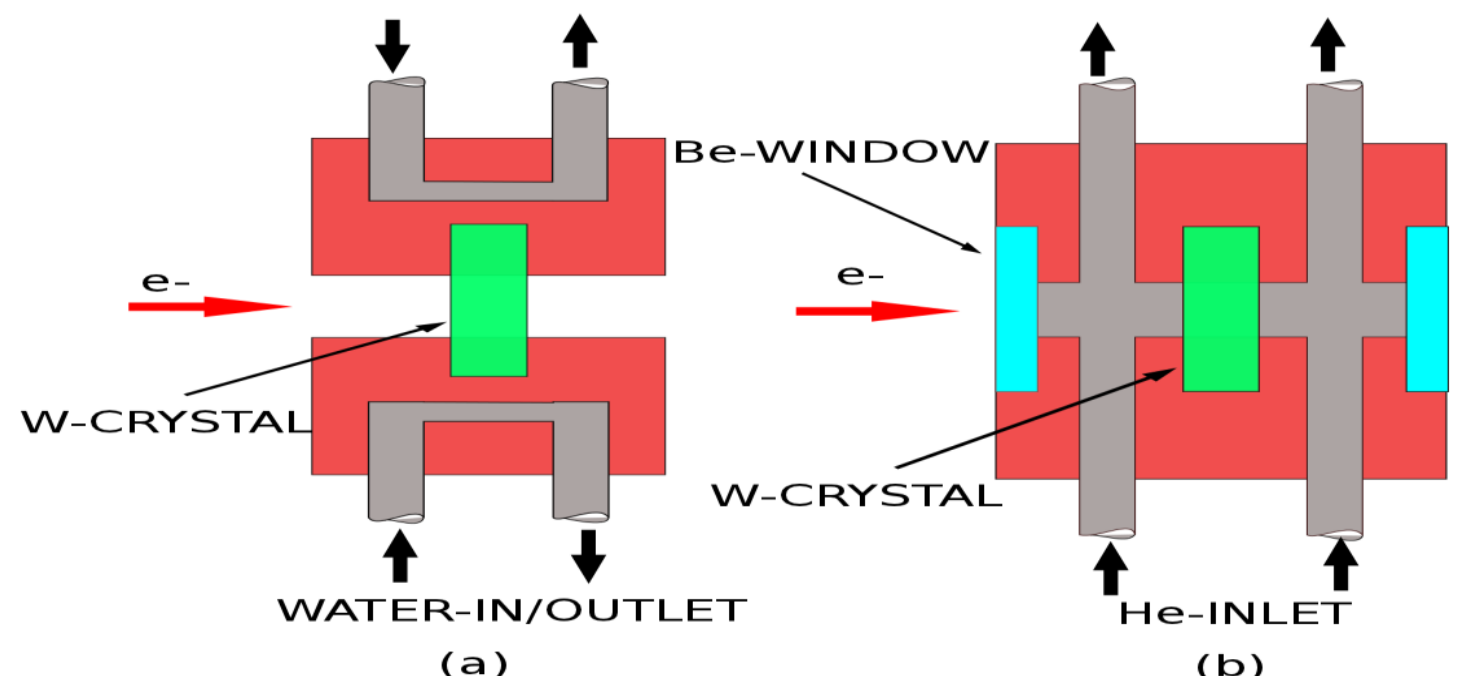

Fig. 9 The crystal mounting with the two cooling options : water cooling and He jet cooling

The amorphous converter is the target where the energy deposition is very important due to the large number of secondaries. For the chosen system $(300 \mathrm{~Hz})$ we have determined the energydeposition density in the (x,z)plane; where $\mathrm{z}$ is the propagation axis. The spheres have $1 \mathrm{~mm}$ radius and correspond to a volumeof $4 \mathrm{~mm}^{3}$ The largest value (PEDD)is 1.8 $\mathrm{GeV} / \mathrm{cm}^{3} / \mathrm{e}-$; it is slightly lowerthan that of a compact target. The PEDD corresponding to a minitrain is about $30 \mathrm{~J} / \mathrm{g}$. The total energy deposited in thetarget is $\sim 446 \mathrm{MeV} / \mathrm{e}-$ for the 3 layers target. The deposited energy density is presented on figure 10 . The vertical scale is in $\mathrm{GeV} / \mathrm{cm}^{3} / \mathrm{e}$-. 


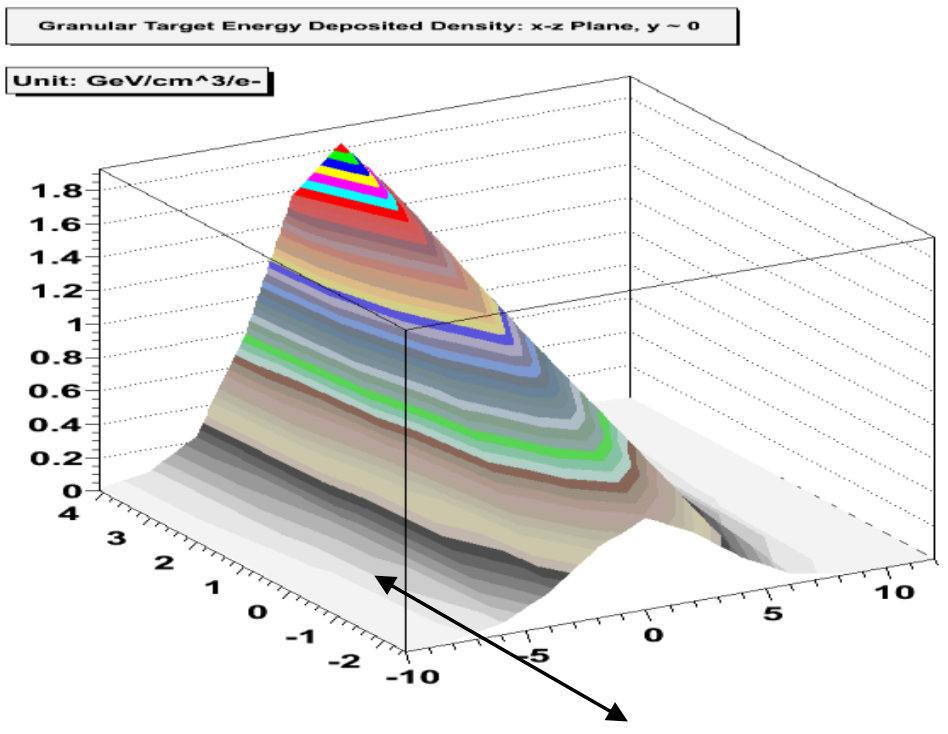

Fig. 10 Energy deposition density in the 3 layers granular converter. Beam direction is indicated ( $\mathrm{z}$ axis). Maximum density is at target exit.

\section{4- HEATING AND COOLING FOR THE GRANULAR CONVERTERS}

In granular targets, consisting of densely packed spheres with sizes smaller than the rmstransverse beam profile of $3.5 \mathrm{~mm}$, only small and essentially constant temperature gradients will be created across each individual sphere. Henceforth,the spheres will be able to expand relatively freely and will be submitted to negligible thermal stresses.

Thermally induced shocks can be neglected when: $r / 2<<t_{0} . c ; r$ : radius of the sphere of $1 \mathrm{~mm}$, to: pulse duration of a micro pulse of 0.6 micro seconds; c: velocity of sound in Tungsten: $\mathrm{c}=4.10^{3} \mathrm{~m} / \mathrm{s}$. For ILC this is satisfied in good approximation.

Another important feature of the granular target is that the deposited heat can be evacuated rapidly and at the location of heat deposition by the cooling fluid passing between the spheres. Excluding a stationary target, two solutions have been considered:

$$
\text { - the rotating wheel }
$$

An overview of the thermal problems associated to this kind of e+ source is found in [17].

\section{4-1 A granular target on a rotating wheel}

A stationary target would receive a too large amount of deposited energy, leading to serious heating problems. To reduce the adiabatic temperature rises induced by one macro pulse, the beam energy can be diluted by sweeping, rotating the target. Since the deposited beam energy is concentrated withina diameter of about $1 \mathrm{~cm}$ ( see previous plot), with a linear velocity of the rim of the rotating wheel of about $3 \mathrm{~m} / \mathrm{s}$, the rim is displaced by $1 \mathrm{~cm}$ over $3.3 \mathrm{~ms}$, so that the energy of each minitrain is separated from the adjacent, following minitrain. Only very little pile up occurs. The calculated maximum temperature rise in the target rim, resulting from the PEDD per minitrain, is not exceeding $222 \mathrm{~K}$. Details on the wheel and on the target container (Be and $\mathrm{Ti}$ ) are presented below.

Beryllium $1 \mathrm{~mm}$ thick is chosen for entrance and exit windows; Titanium $1 \mathrm{~mm}$ thick is chosen for upper and lower parts of the container. Energy deposited and PEDD have been evaluated for the windows; they are negligible for Ti, whereas for Be the PEDD represents $30.6 \mathrm{~J} / \mathrm{g}$ (downstream window) leading to sustainable temperature rise of about $15^{\circ} \mathrm{K} / \mathrm{minitrain}$. The rotating wheel is represented on figure 11.To minimize the temperature rise in the Helium during its passage along the periphery, its path length should be minimized by increasing the number of spokes for the He inlets and outlets. 


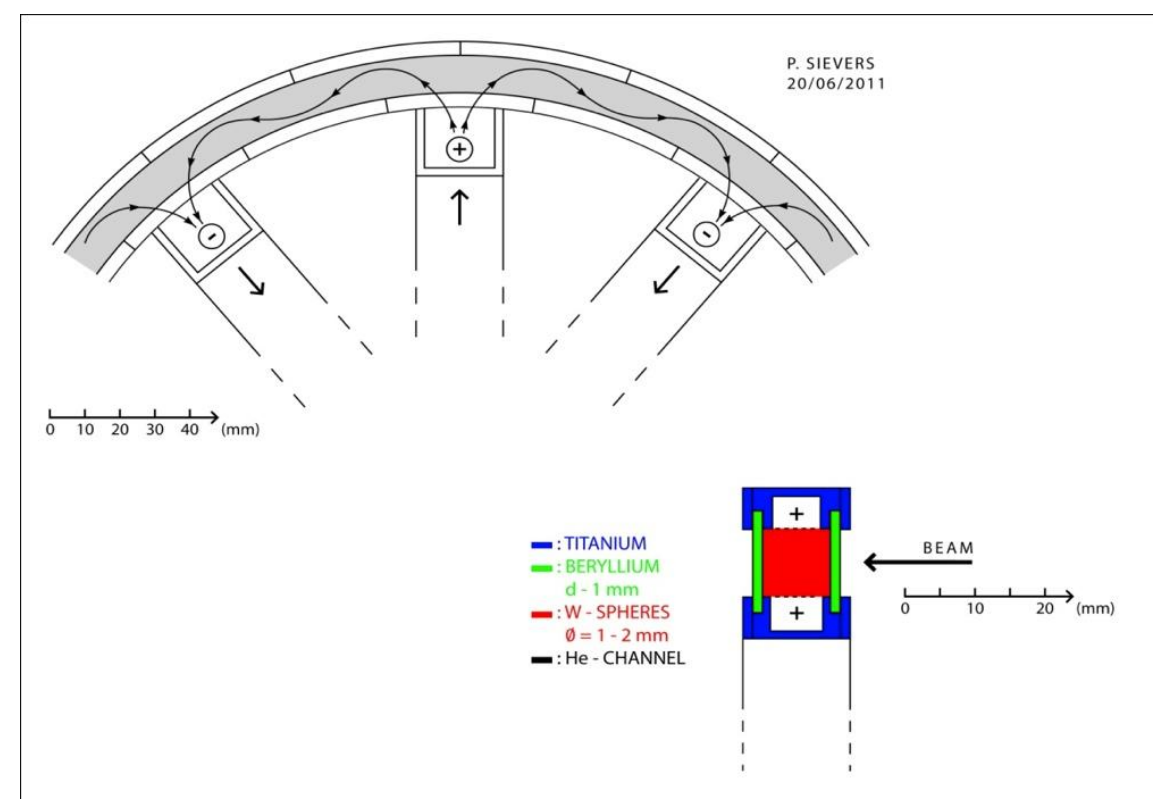

Fig. 11The rotating wheel

The minimum periphery of the rotating wheel would be $13 \mathrm{~cm}$, i.e. a diameter of $4 \mathrm{~cm}$, to distribute uniformly a macro pulse over its rim over $40 \mathrm{~ms}$. This results in a rotation frequency of $23 \mathrm{~Hz}$ or $1400 \mathrm{rpm}$, which may be difficult to achieve with rotating seals for the He inlets and outlets..

Therefore a larger wheel with a diameter of $58 \mathrm{~cm}$ is considered. With a velocity of the rim of $3.25 \mathrm{~m} / \mathrm{s}$, allowing for comfortable separation between minitrains, this leads to a rotation at $1.786 \mathrm{~Hz}$ or 107 r.p.m. which will be much easier to engineer. $3.10^{6}$ pulses will be accumulated per target over 100 days of operation. With this configuration and taking into account the repetition frequency of the macro pulses of $5 \mathrm{~Hz}$, it results that, in the average, each sphere will be hit every 5 turns, i.e. every $2.8 \mathrm{~s}$. The total, average power, to be removed from a wheel (as from a stationary target) is about $10 \mathrm{~kW}$. With Helium, pressurized to $1 \mathrm{MPa}$ and with an entrance velocity of $10 \mathrm{~m} / \mathrm{s}$, a He mass flow of $30 \mathrm{gr} / \mathrm{s}$ is required which would result in an average temperature rise of $84{ }^{\circ} \mathrm{C}$ at the exit of the He-flow. Temporary and locally, values of $250{ }^{\circ} \mathrm{C}$ in the He may, however, occur at the hottest sphere just after a minitrain. Clearly, the wheel must be made vacuum tight and resist to the internal He-pressure of $1 \mathrm{MPa}$. Moreover, with the above specified He-cooling, the time constant of the exponential decrease in temperature of an adiabatically heated sphere is about $100 \mathrm{~ms}$. Thus, a sphere will be cooled to practically zero before being hit again after $2.8 \mathrm{~s}$.

4-2 Eddy currents: interference of the magnetic fringe field of the flux concentrator with the adjacent rotating wheel has been studied for fast rotating wheels (I.Bailey). With the above velocity $\sim 3 \mathrm{~m} / \mathrm{s}$, this problem should be much reduced. Anyway, this must be confirmed by further studies.

\section{4-3-The pendulum}

To avoid rotating seals, wobbling or trolling targets have been devised at Durham ILC e+ Meeting in October 2009, where the displacement of the target structure from the outside into the vacuum is made via flexible, vacuum tightbellows. The injection ofthe cooling fluid 
can thus be ensured through a rigid, non rotating structure. Inthe following we consider a "Pendulum Target", where the required displacement and velocity is provided by the sinusoidal oscillation of the target (figure 12). The width of the target is $13 \mathrm{~cm}$, providing space for 13 minitrains with a diameter of $1 \mathrm{~cm}$ each and being displaced, when the beam is hitting the target, at a velocity of about $3 \mathrm{~m} / \mathrm{s}$ over $+/-7.5$ degrees. The total swing is $+/-23$ degrees, allowing for comfortable inversion of the direction of the movement during the "off beam" time of $160 \mathrm{~ms}$. The same range in angle must be sustained by the bellows oscillating at $2.5 \mathrm{~Hz}$. Since in this configuration the average time between hits of the same target spot is about $0.2 \mathrm{~s}$ as compared to the rotating wheel with $2.8 \mathrm{~s}$, average temperatures higher by about $100 \mathrm{~K}$ will result. This might, if necessary, be compensated by an improved He cooling. Clearly, prototyping will be required, also to assess the life time of the spheres, the windows and the bellows, submitted to $4.10^{7}$. cycles over 100 days of continuous operation.

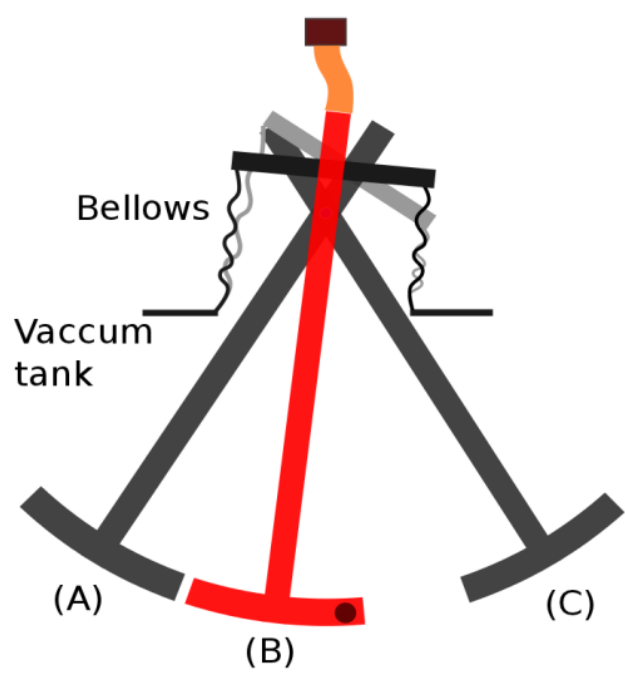

(a)

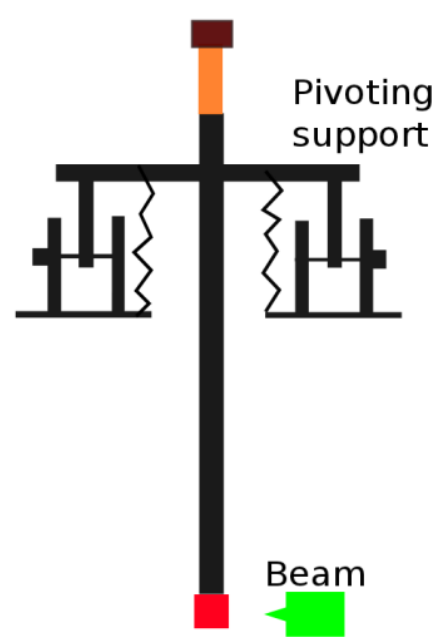

(b)

Fig. 12The pendulum for a granular target

\section{5- SUMMARY AND CONCLUSIONS}

The hybrid target with a granular amorphous converter exhibits:

- - a good quality positron beam (yield, emittance) generated by photons from channeling radiation

- the PEDD for the granular converter is lower than for the compact one

- an efficient thermal dissipation process due to the granular character.

In particular, the enlargement of the macro pulse to $40 \mathrm{~ms}$ duration in the above considered « $300 \mathrm{~Hz}-\mathrm{KEK}-\mathrm{System} »$ and leaving a gap of «no-beam » of $160 \mathrm{~ms}$, sufficient for efficient damping, opens the possibility to reduce the thermal load from the macro pulse on the target by displacing it laterally through rotation or trolling it. Lateral velocities of about $3 \mathrm{~m} / \mathrm{s}$ for this displacement are sufficient to reduce the local energy deposition density by a factor of 13 (in one macropulse). Using granular targets, consisting of an ensemble of Tungsten spheres of $2 \mathrm{~mm}$ diameter or below and cooled by a Helium gas stream passing between the spheres, tolerable temperatures for Tungsten, Helium and the target container are achieved. In addition to a rotating wheel, a pendulum target is considered, by which the use of rotating vacuum and He-seals is avoided. Both target types seem to represent viable solutions for the positron production in the considered ILC scheme. 


\section{Acknowledgements}

The authors are indebted to T.Suwada, T.Kamitani, T.Omori, J.Urakawa (KEK), T.Takahashi (Hiroshima University), L.Rinolfi, A.Vivoli (CERN) for valuable discussions. Thanks also to B.Mazoyer (LAL) for his help.

\section{REFERENCES}

[1] V.N. Baier, V.M. Katkov, V.M. Strakhovenko "Radiation yield of high-energy electrons in thick crystals" Phys.Stat.Sol. (b) 133, (1986) 583-592

[2] X.Artru, V.N.Baier, R.Chehab, A.Jejcic "Positron source using channeling in a tungsten crystal" Nucl.Instrum.Methods A 344 (1994) 443-454

[3] X.Artru et al. "Axial channeling of relativistic electrons in crystals as a source for positron production" Nucl.Instrum.Methods B 119 (1996) 246-252

[4] X.Artru, R.Chehab, M.Chevallier, V.M.Strakhovenko "Advantages of axially aligned crystal in positron production at future linear collider" Phys. Rev. Special Topics A-B 6 (2003) 091003

[5] R.Chehab et al. "Experimental study of a crystal positron source" Physics Letters B 525 (2002) 41-48

[6] X.Artru et al. "experiment with a crystal-assisted positron source using 6 and $10 \mathrm{GeV}$ electrons" Nucl.Instrum.Methods B 201 (2003) 243-252

[7] X.Artru et al. "Summary of experimental studies at CERN on a positron source using crystal effects" Nucl.Instrum.Methods B 240 (2005) 762-

[8] T.Suwada et al. "Measurement of positron production efficiency from a tungsten monocrystal using 4 and 8 GeV electrons" Phys. Rev. E 67 (2003) 016502

[9] T.Suwada et al. "First application of a tungsten single crystal positron source at the KEKB factory" Phys. Rev. Special Topics A-B 10 (2007) 073501

[10] X.Artru, R.Chehab, M.Chevallier, V.M.Strakhovenko, A.Variola, A.Vivoli “ Polarized and unpolarized positron sources for electron-positron colliders" Nucl.Instrum.Methods B 266 (2008) 3868-3875

[11] X.Artru et al "Positron sources using channeling: a promising device for linear colliders" Il NuovoCimento C 34 N.4 (July-August 2011)

[12] P.Sievers, P.Pugnat“a He-gas cooled stationary target” J.Phys. G: Part.Phys. 29 (2003) $1797-1800$

[13] R.Chehab et al. Proc. IEEE Part.Accel.Conf., Chicago, IL, March 1989

[14] V.M.Strakhovenko (programme VMS)

[15] T.Omori (see POSIPOL 2009 and POSIPOL 2010)

[16] R.Chehab et al. "Radiation damage study of a monocrystalline tungsten converter" in Proceedings of EPAC'98, Stockholm, 1998 and 1AL-RT 98-02

[17] P.Sievers et al. "Positron Source using channeling with a granular converter" in Proceedings of POSIPOL 2011 Workshop, August 2011, Beijing (China). 